\title{
Validation of the Actical and Actiheart monitor in ambulatory children with Spina Bifida
}

\author{
J.F. de Groot ${ }^{\mathrm{a}, \mathrm{b}, *}$, A.S. de Jong ${ }^{\mathrm{c}}$, T. Visser ${ }^{\mathrm{c}}$ and T. Takken ${ }^{\mathrm{b}}$ \\ ${ }^{a}$ Research Group Lifestyle and Health, University of Applied Sciences, Utrecht, The Netherlands \\ ${ }^{\mathrm{b}}$ Child Development and Exercise Center, Wilhelmina Children's Hospital, University Medical Center Utrecht, \\ Utrecht, The Netherlands \\ ${ }^{\mathrm{c}}$ Faculty of Human Movement Sciences, Vrije University Amsterdam, Amsterdam, The Netherlands
}

Accepted for publication 15 April 2013

\begin{abstract}
.
BACKGROUND: Ambulatory children with Spina Bifida (SB) often show a decline in physical activity leading to deconditioning and functional decline. Therefore, assessment and promotion of physical activity is important. Because energy expenditure during activities is higher in these children, the use of existing pediatric equations to predict physical activity energy expenditure (PAEE) may not be valid.

AIMS: (1) To evaluate criterion validity of existing predictions converting accelerocounts into PAEE in ambulatory children with SB and (2) to establish new disease-specific equations for PAEE.

METHODS: Simultaneous measurements using the Actical, the Actiheart, and indirect calorimetry took place to determine PAEE in 26 ambulatory children with SB.

DATA ANALYSIS: Paired T-tests, Intra-class correlations limits of agreement (LoA), and explained variance $\left(\mathrm{R}^{2}\right)$ were used to analyze validity of the prediction equations using true PAEE as criterion. New equations were derived using regression techniques.

RESULTS: While T-tests showed no significant differences for some models, the predictions developed in healthy children showed moderate ICC's and large LoA with true PAEE. The best regression models to predict PAEE were: PAEE = 174.049 $+3.861 \times$ HRAR $-60.285 \times$ ambulatory status $\left(R^{2}=0.720\right)$ and PAEE $=220.484+0.67 \times$ Actical counts $-60.717 \times$ ambulatory status $\left(\mathrm{R}^{2}=0.681\right)$.

CONCLUSIONS: Existing equations to predict PAEE are not valid for use in children with SB for the individual evaluation of PAEE. The best regression model was based on HRAR in combination with ambulatory status, followed by a new model for the Actical monitor. A benefit of HRAR is that it does not require the use of expensive accelerometry equipment. Further cross-validation of these models is still needed.
\end{abstract}

Keywords: Accelerometry, Spina Bifida, child, physical activity

* Corresponding author: J.F. de Groot, Research Group Lifestyle and Health, HU University of Applied Sciences Utrecht, and Child Development and Exercise Center, Wilhelmina Children's Hospital, University Medical Center Utrecht, Room kb.02.056.0, P.O. Box 85090, 3508 AB Utrecht, The Netherlands. Tel.: +31 88755 4030; Fax: +31 88755 5333; E-mail: Janke.degroot@hu.nl.

\section{Introduction}

Spina Bifida (SB) is the most frequent congenital deformity of the neural tube, with incidence ranging from 3-4 new cases per 10,000 births in most industrialized countries, while in some other countries, such as Ukraine, France, Germany, Malta, Japan, and India, these numbers range from 7-12.8 [1]. Depend- 
ing on both type and level of the SB lesion, patients can experience a variety of deficits in cognition, motor function, sensory function, and bowel and bladder function [2]. Due to advances in the medical approach, mortality rates have decreased in recent years and $60-80 \%$ of children with SB can now be expected to live to be healthy adults [3-6]. This requires a different approach to the medical management of these patients from childhood through adolescence and adulthood, one that focuses not only on the pathological aspects, but also on the preventable medical and social consequences of the disease [7].

As a result of reduced mobility, adolescents and young adults are at risk of developing a negative spiral deriving from an inactive lifestyle [8], which may lead to obesity, risk factors for cardiovascular disease, reduced quality of life, and even wheelchair dependence [9]. In addition, Van den Berg-Emons et al. [8] reported that physical activity (PA) levels in adolescents and young adults with SB (particularly nonambulatory) were considerably lower when compared to healthy peers. In this study, levels of objectively measured PA seemed to be significantly related to ambulation level and fitness. In children with SB, studies have reported lower levels of physical activity, but these levels were assessed either by subjective recall in a diary [10] or expensive methods such as doublylabelled water [11]. In order to gain more insight into daily physical activity levels in ambulatory children with SB and how this relates to physical fitness, the use of objective and mobile systems is recommended [10, 12], though currently not available.

Different techniques can be used for the acquisition of data on postures and motions in daily living, such as questionnaires, diaries, gyroscopes, pedometers, accelerometers, or heart rate monitors. Each technique has its own benefits and limitations when objectively measuring physical activity energy expenditure (PAEE) performed throughout the day. In healthy children several accelerometers have been proven to be a valid instrument to accurately estimate PAEE during activities of daily living [13-15]. One of them is the Actical (Philips Respironics, Mini Mitter Co, Inc., Bend, OR), which records both duration and intensity of PAEE performed throughout the day. At the same time, the Actical seems less accurate for detecting changes in more energy-consuming gait patterns [16]. For this reason, heart rate (HR) could better distinguish between different types of walking, as it has the advantage of being a physiological variable that is directly related to oxygen uptake $\left(\mathrm{VO}_{2}\right)$. HR by itself, however, can be elevated due to other factors, such as stress, dehydration, and environmental factors, e.g. temperature [17]. Therefore, a combination of the two techniques can lead to improved estimation of PAEE. The Actiheart (Cambridge Neurotechnology, Cambridge, UK) is a commercially available device that combines heart rate monitoring and accelerometry into a single unit that has also be proven suitable for use with healthy children [13,14].

Earlier studies have shown higher levels of PAEE during ambulation in patients with SB. Higher levels of PAEE are associated with a pathological gait pattern due to muscle weakness in the lower extremities [1820]. With different energy expenditure and locomotion patterns, the question is whether existing PAEE equations developed in healthy children are valid for ambulatory children with SB. Thehypothesis of this study is that due to the higher energy cost of locomotion, existing PAEE equations underestimate the true PAEE in patients with SB for two reasons. Firstly, the different gait pattern may result in a different registration of activity counts. Secondly, more energy expenditure per activity count might result in an underestimation of true PAEE during daily activities. In a more recent publication, new prediction models have been proposed for children with chronic disease [21]. However, this study did not include populations known to have different patterns of energy expenditure during locomotion, as children with SB do. The second hypothesis of the current study is that the prediction models for children with chronic disease would underestimate energy expenditure in children with SB as well.

Therefore, the first aim of this study was to test the hypotheses that prediction equations derived by Corder et al. [13,14] and those by Takken et al. [21] used to estimate PAEE in children are not valid in ambulatory children with SB. Secondly, if this hypotheses turned out to be correct, the next aim was to develop new regression models to predict the PAEE in ambulatory children with SB.

\section{Methods}

\subsection{Study population}

This study was part of a larger study (the Utrecht Spina Bifida And Graded Exercise (USAGE) study) regarding exercise and physical activity in ambulatory children with SB [22]. Study procedures took place at the Child Development and Exercise Center of the 
Table 1

Ambulation level by Hoffer et al., adapted by Schoenmakers et al. [24]

\begin{tabular}{ll}
\hline Level of ambulation & Description \\
\hline Normal ambulation & Independent and unrestricted ambulation without use of assisted devices \\
Community ambulation & Independent outdoor ambulation with or without use of braces and/or assisted devices; using wheelchair for \\
& longer distances \\
Household ambulation & Using braces or assisted devices for indoor ambulation; using wheelchair for outdoor locomotion \\
Non-functional ambulation & Walking only in therapeutic situations \\
Non-ambulation & Wheelchair dependent \\
\hline
\end{tabular}

Wilhelmina Children's Hospital. All study procedures were approved by the local Medical Ethics Committee. Parents and children signed informed-consent forms prior to testing.

Children were included when they were: (1) at least community ambulatory according the adapted Hoffer scale $[23,24]$; (2) able to follow instructions regarding testing; (3) willing and able to correctly wear the physical activity monitor for three consecutive days; (4) between six and 18 years of age; and (5) when complete data for all measurements was available.

\subsection{Demographics and disease specific variables}

Data concerning medical history were obtained from medical records. These data included the level of lesion, the ambulation level, age, and sex.

\subsection{Anthropometrics}

Weight $(\mathrm{kg})$ was measured using an electronic scale. Height (m) was measured while standing using a wallmounted stadiometer. Body mass index (BMI) was calculated as weight $(\mathrm{kg}) /$ height $(\mathrm{m})^{2}$.

Body composition was assessed using the sum of seven skin folds according to Pollack et al. [25]. The measurements were taken with a Harpenden skin fold caliper at the biceps, triceps, supra-iliacal, midabdominal, sub scapular, medial thigh, and calf, at the right and left side of the body.

Ambulatory function was rated on the adapted Hoffer scale [23,24], see Table 1.

\subsection{Test protocol}

During a six-minute walk test (6MWT), study participants were simultaneously wearing an Actical, an Acitheart, and a mobile gas-analysis system (indirect calorimetry). In doing so, PAEE as predicted by the accelerometers could be compared to PAEE as measured by indirect calorimetry during the same period.

\subsubsection{Indirect Calorimetry (IC)}

During rest and ambulation, physiologic responses were measured using a heart rate monitor (Polar) and calibrated mobile gas analysis system (Cortex meta$\max \mathrm{B}^{3}$, Cortex Medical GmbH, Leipzig, Germany) for breath-by breath analysis. All measurements and calibrations were performed according to the manufacturer's instructions and guidelines. The mask was checked for possible leakage before the test. The Cortex Metamax is a valid and reliable system for measuring gas-exchange parameters during exercise [26,27].

Resting measurements were recorded while the participants were sitting reading a comic book for $10 \mathrm{~min}$ utes. PAEE was measured during a 6MWT. The 6MWT test was chosen for two reasons: (1) an earlier study had shown it is possible to reach a steady state of $\mathrm{VO}_{2}$, which is a prerequisite for estimating EE [28], and (2) ambulation was the most commonly cited way of being active in ambulatory children with SB (unpublished report). The test was performed on a 13-meter track in a straight corridor and not on the recommended 30-meter track, since this was not feasible in the testing facility used. Participants were instructed to cover the largest possible distance in six minutes at a self-selected walking speed. The encouragements during the test were performed in accordance with the American Thoracic Society guidelines [29].

\subsubsection{Energy expenditure analysis}

Steady State (SS) normalized oxygen uptake $\left(\mathrm{O}_{2}\right.$. $\mathrm{kg}^{-1} \cdot \mathrm{min}^{-1}$ ) was calculated as the average value over the period during which oxygen uptake changed not more than $5 \%$. For this purpose, $\mathrm{VO}_{2}$ was plotted for visual inspection. Within the period of least differences, a SS of two minutes was determined. Respiratory exchange ratio (RER) was calculated as $\mathrm{VCO}_{2} / \mathrm{VO}_{2}$ during steady state. Resting energy expenditure (REE) and gross energy consumption ( $\mathrm{EE}_{\text {gross }}$ ) were subsequently derived. Physical activity energy expenditure (PAEE) was calculated as $\mathrm{EE}_{\text {gross }}$ minus REE. PAEE was expressed in $\mathrm{J} \cdot \mathrm{kg}^{-1} \cdot \mathrm{min}^{-1}$, using $\mathrm{VO}_{2}$ and RER in the following equation: $\mathrm{J} \cdot \mathrm{kg}^{-1} \cdot \mathrm{min}^{-1}$ $=(4.960 \times$ RER during steady state +16.040$) \times$ $\mathrm{VO}_{2} \cdot \mathrm{kg}^{-1}[30]$. 


\subsubsection{Heart rate measures}

Heart rate above rest (HRAR)

HRAR was calculated as HR during the steady state of walking minus resting HR. Resting HR was taken from the resting measurements (see above) prior to the 6MWT.

\section{Heart rate above sleep (HRAS)}

For HRAS, both real HRAS and predicted HRAS were calculated, because existing prediction equations often use predicted HRAS, while in this study real measured HRAS was available. HRAS was calculated as HR during the steady state activity minus HR during sleep. For heart rate during sleep, the $10^{\text {th }}$ lowest HR observed during sleeping (from 00:00 until 03:00 AM) was taken to obtain a robust estimate of this parameter according to Brage et al. [31].

For the predicted HRAS, predicted heart rate during sleep was estimated using the following equation by Corder et al. [13]. Predicted heart rate during sleep = $0.4195 \times$ resting heart rate +27.4 . Predicted HRAS was then calculated as HR during steady state minus predicted HR during sleep.

\subsubsection{Activity monitors}

Actical (AC)

The $\mathrm{AC}$ is a compact $(28 \times 27 \times 10 \mathrm{~mm})$ and lightweight (17 g) omni directional accelerometer that records accelero counts, further referred to as "acticounts". It is sensitive to movements in the $0.5-$ to $3-\mathrm{Hz}$ range. It is most sensitive to vertical accelerations of the torso when placed on the hip $(31 ; 13)$. The AC was mounted on an elastic waist strap and placed at the left hip of the participants. The AC monitors the amount and intensity of motion. The AC was set to record data in 1 minute epochs.

\section{Actiheart (AH)}

The $\mathrm{AH}$ is a compact $(7 \times 33 \mathrm{~mm})$ and lightweight $(8 \mathrm{~g})$ device that records acticounts and HR. A detailed description is available elsewhere [16]. Briefly, the $\mathrm{AH}$ was placed with two standard electrodes $\left(3 \mathrm{M}^{\mathrm{tm}}\right.$ Red Dot ${ }^{\mathrm{tm}} 2271$ Monitoring Electrodes, 3M Nederland BV, Zoeterwoude, the Netherlands) on the participants chest. The $\mathrm{AH}$ is able to measure acceleration, HR, HR variability, and ECG magnitude for epoch settings of 15, 30, and 60 seconds (s). Data is recorded with a memory capacity of 128 kbytes. Acceleration is measured by a piezoelectric element with a frequency of $1-7 \mathrm{~Hz}(3 \mathrm{~dB})$. AH monitors were calibrated by the manufacturer. The monitor was set to record data in 15 s epochs.

\subsection{Data analyses for activity monitor data}

First, the measurement data from the activity monitors was downloaded using a docking station and proprietary software. It was then exported to a spread sheet that showed both the activity counts and HR.

Data from the AC was converted in PAEE using a prediction equation for healthy children by Corder et al. [13]:

- Actical hip (AC): PAEE $\left(\mathrm{J} \cdot \mathrm{kg}^{-1} \cdot \mathrm{min}^{-1}\right)=0.2$ acticounts +168.7

Data from the AH was converted in PAEE using three prediction equations by Corder et al. [13,14]:

- Actiheart Activity Counts (AH1): PAEE (J.kg-1. $\left.\min ^{-1}\right)=0.22$ acticounts +29.3 gender +144.3

- Actiheart Activity HR (AH2): PAEE $\left(\mathrm{J} \cdot \mathrm{kg}^{-1}\right.$. $\left.\min ^{-1}\right)=6.1$ HRAR -6.7 gender $+1.4($ gender $\times$ HRAR) -12.1

- Actiheart Activity Combined (AH3): PAEE (J. $\left.\mathrm{kg}^{-1} \cdot \mathrm{min}^{-1}\right)=4.4$ HRAR +0.08 acticounts 2.7 gender +1.1 (gender $\times$ HRAR $)+15.1$

- Actiheart Activity Combined (AH4): PAEE (J. $\left.\mathrm{kg}^{-1} \cdot \mathrm{min}^{-1}\right)=5.17 \times$ predicted HRAS +0.61 $\times$ gender $\times$ HRAS $+0.07 \times$ acticounts $-0.6 \times$ gender $-74($ girls $=0$; boys $=1)$

Data for the AH was converted in PAEE using the following prediction for children with chronic disease [21]:

- Actiheart (Takken): PAEE $=-93.7+4.8 \times$ HRAS $+0.04 \times$ acticounts $+39.8 \times$ gender (girls $=0$; boys $=1$ )

In predictions equations using HRAS (AH4 and Takken), both real HRAS and predicted HRAS were entered.

\subsection{Statistical analyses}

\subsubsection{Part I: Validity of prediction equations}

All variables were checked for normal distribution using the Kolmogorov-Smirnov test and visual inspection of Q/Q plots. For descriptive purposes, parametric descriptive statistics were used (mean \pm SD). Paired samples T-tests were carried out to check for differences in (1) predicted HRAS and actual HRAS and (2) PAEE and the predictions by Corder and Takken.

For validity of the predicted PAEEs derived from Corder et al. [13,14] and Takken et al. [21], intra-class coefficients (ICC) were computed to explore the relationship between predicted PAEE and the PAEE derived from the indirect calorimetry. An ICC of 0.8 or 
Table 2

Participant characteristics

\begin{tabular}{|c|c|c|c|}
\hline & $\begin{array}{c}\text { All children }(n=26) \\
\text { Mean }(\mathrm{SD})\end{array}$ & $\begin{array}{c}\text { Normal ambulatory children } \\
(n=7) \text { Mean (SD) }\end{array}$ & $\begin{array}{c}\text { Community ambulatory children } \\
(n=19) \text { Mean (SD) }\end{array}$ \\
\hline Age (years) & $10.6(2.8)$ & $11.9(3.5)$ & $10.1(2.4)$ \\
\hline Weight (kg) & $43.9(21.1)$ & $45.7(19.1)$ & $43.3(22.3)$ \\
\hline Height $(\mathrm{cm})$ & $141.8(18.6)$ & $149.7(18.1)$ & $138.9(18.4)$ \\
\hline BMI & $20.6(5.3)$ & $19.4(3.5)$ & $21.0(5.8)$ \\
\hline Sum skinfolds (mm) & $115.9(62.9)$ & $102.8(46.9)$ & $120.8(68.3)$ \\
\hline Resting energy expenditure $\left(\mathrm{J} \cdot \mathrm{kg}^{-1} \cdot \mathrm{min}^{-1}\right)$ & $155.0(59.3)$ & $146.6(66.1)$ & $158.1(58.2)$ \\
\hline Resting heart rate & $86.4(14.1)$ & $81.0(14.3)$ & $88.4(13.9)$ \\
\hline Acticounts actical during ambulation & $2547.9(1024.9)$ & $2983.1(1168.0)$ & $2387.6(950.5)$ \\
\hline Acticounts actiheart during ambulation & $857.3(394.3)$ & $1153.9(311.3)^{*}$ & $748.0(369.0)$ \\
\hline
\end{tabular}

Legend: $\mathrm{SD}=$ Standard deviation; BMI = Body Mass Index; $*=$ significant difference $(p=0.015)$; $=$ Joules; $1 \mathrm{~J}=0.239$ Calories.

Table 3

ICC, T-test, and Limits of Agreement (LoA) of predicted PAEE in 26 ambulatory children with SB

\begin{tabular}{|c|c|c|c|c|c|}
\hline Gold standard (J.kg $\left.{ }^{-1} \cdot \mathrm{min}^{-1}\right)$ & Predictions $\left(\mathrm{J} \cdot \mathrm{kg}^{-1} \cdot \mathrm{min}^{-1}\right)$ & Difference $*(p)$ & $\mathrm{ICC}(\mathrm{CI})$ & LoA & $\mathrm{R}^{2}$ \\
\hline PAEE 347.05 (97.5) & AC $678.3(205.0)$ & $-331.2(0.000)^{* *}$ & $0.60(0.28-0.80)$ & $+616.70 /-45.77$ & 0.61 \\
\hline PAEE 347.05 (97.5) & AH1 349.8 (86.4) & $-2.7(0.88)$ & $0.49(0.12-0.74)$ & $+189.12 /-183.6$ & 0.24 \\
\hline PAEE $344.1(98.3)$ & AH2\# 380.9 (148.7) & $-33.8(0.22)$ & $0.74(0.49-0.88)$ & $+243.72 .2 /-176.1$ & 0.59 \\
\hline PAEE 347.105 (98.3) & АH3\# 371.04 (121.0) & $-25.0(0.26)$ & $0.80(0.59-0.91)$ & $+180.72 /-132.74$ & 0.65 \\
\hline PAEE 347.105 (97.5) & AH4 predHRAS 337.07 (48.1) & $10.0(0.62)$ & $0.12(-0.28-0.48)$ & $+194.14 /-214.10$ & 0.02 \\
\hline PAEE\# 347.05 (97.5) & Takken predHRAS 269.02 (38.7) & $78.03(0.001)^{* *}$ & $0.018(-0.37-0.40)$ & $+129.81 /-285.87$ & 0.0007 \\
\hline PAEE 347.105(97.5) & AH4 true HRAS 430.4 (135.8) & $-83.3(0.000)^{* *}$ & $0.71(0.45-0.86)$ & $+263.11 /-96.42$ & 0.56 \\
\hline PAEE $347.105(97.5)$ & Takken true HRAS 351.8 (113.4) & $-4.7(0.76)$ & $0.73(0.48-0.87)$ & $+159.46 /-149.96$ & 0.55 \\
\hline
\end{tabular}

Legend: *T-test; ICC- intra-class correlation; $\mathrm{CI}=$ Confidence Interval; LoA $=$ Limits of Agreement; $R^{2}=$ explained variance; $* *=$ significant; $\mathrm{PAEE}=$ Physical Activity Energy Expenditure; HRAS = heart rate above sleep; $\mathrm{AC}=$ Actical; $\mathrm{AH}=$ Actiheart; \#outlier removed; $\mathrm{J}=\mathrm{J}$ oules; $1 \mathrm{~J}=0.239$ Calories.

higher was considered good [32]. A p-value of less than 0.05 was used to determine statistical significance.

Subsequently, Bland-Altman plots were used to check the degree of heteroscedasticity and the limits of agreement (LoA) between the predicted PAEE and the real PAEE measured by IC. The closer the mean is to zero and the smaller the standard deviations, the better the agreement [33]. Explained variance $\left(\mathrm{R}^{2}\right)$ was calculated in order to be compare these models with the new regression models.

\subsubsection{Part II: New regression models}

First, correlation coefficients were computed to explore the relationship between anthropometric, physiological, and disease-related variables and the main outcome parameter PAEE. Variables with significant correlations of $>0.5$ were entered into the regression model to predict PAEE. At the same time, these correlations were used to check for possible co-linearity. When co-linearity was present, only the highest correlate would enter the stepwise regression. T-tests were carried out to test differences in PAEE between girls and boys and between normal and community ambulatory children. The regression models were evaluated by significance differences in the sum of least squares
(F-statistic). Only significant models were evaluated for the highest explained variance $\left(\mathrm{R}^{2}\right)$ in prediction of PAEE.

Statistical analyses were performed using SPSS for Windows (version 17.0, SPSS Inc., Chicago, Ill).

\section{Results}

\subsection{Population}

The study population came from a database consisting of 39 ambulatory children with SB. Participants were included in the study when data from both the indirect calorimetry, the $\mathrm{AC}$, and the $\mathrm{AH}$ where complete. Data of 13 participants were not taken into account due to missing data and software/hardware difficulties of the IC, AC or AH. This resulted in a convenience sample of 26 ambulatory children (15 boys/ 11 girls) with SB. Data were distributed normally. The characteristics of the participants are shown in Table 2 . According to the adapted classification for functional ambulation, seven children were normal ambulatory and 19 children were community ambulatory. There were no significant differences for anthropometric measurements and resting measures between these two groups. 
Table 4

Pearson correlations between Physical Activity Energy Expenditure (PAEE) and anthropometric and disease-related variables and acticounts

\begin{tabular}{lc}
\hline & PAEE pearson correlation $(p$ value $) n=26$ \\
\hline Age & $0.211(0.323)$ \\
HR rest & $-0.056(0.789)$ \\
HRAS & $0.725(0.000)^{*}$ \\
HRAS predicted & $-0.107(0.604)$ \\
HRAR $(n=25)$ & $0.805(0.000)^{*}$ \\
BMI & $0.121(0.556)$ \\
Height & $0.291(0.149)$ \\
Weight & $0.198(0.333)$ \\
Sum of skinfolds & $0.163(0.427)$ \\
Level of lesion\# & $0.042(0.842)$ \\
AC counts & $0.779(0.000)^{*}$ \\
AH counts & $0.526(0.006)^{*}$ \\
\hline
\end{tabular}

Legend: $*=$ significant; $\#=$ Spearman correlation; PAEE = Physical Activity Energy Expenditure; HRAS = heart rate above sleep; $\mathrm{HRAR}=$ heart rate above rest; $\mathrm{BMI}=$ Body Mass Index; $\mathrm{AC}=\mathrm{Ac}-$ tical; $\mathrm{AH}=$ Actiheart.

\subsection{Part I: Validity of predicted PAEE}

After the first analysis of the data, one outlier was removed for data concerning HRAR derived measures, due to extreme measures.

Differences between PAEE as measured by indirect calorimetry and predicted PAEE, ICC, LoA, and explained variance can be found in Table 3 .

First of all, a significant difference $(p=0.01)$ of 17.5 beats per minute was noted between HRAS $(80.0$ (23.0)) and predicted HRAS (63.6 (5.9)), with a large difference in the SD as well. Differences between predicted PAEE and actual PAEE were large and significant for the prediction based on Actical prediction. ICC for models using HRAR (AH2 and AH3) and models using real HRAS (AH4 and Takken) showed good ICC's, large LoA, and explained variance ranging from $55 \%$ to $65 \%$. Models using Actiheart acticounts only (AH1) or predicted HRAS (AH4 pred HRAS and Takken pred HRAS) had low ICC's, large LoA, and very low explained variance $(0.07 \%$ to $24 \%)$. Based on these results, new equations were derived.

\subsection{Part II: New prediction equations}

First correlations were calculated between PAEE by IC and anthropometric, physiological, and diseaserelated variables (see Table 4 ).

Significant correlations were present between PAEE and heart rate measures, except for predicted HRAS. At the same time, acticounts from both the $\mathrm{AC}$ and $\mathrm{AH}$ measures correlated significantly with PAEE. These variables were entered into the regression analyses.
Table 5

New PAEE prediction models for children with SB

\begin{tabular}{llc}
\hline Model & Prediction equations & $\mathrm{R}^{2}$ \\
\hline Actical $(n=26)$ & PAEE $=220.484+0.67 *$ AC & 0.68 \\
& counts $-60.717 *$ ambulatory status & \\
Actiheart $(n=25)$ & PAEE $=174.049+3.861 *$ HRAR -0.72 \\
& $60.285 *$ ambulatory status & \\
\hline
\end{tabular}

Legend: PAEE $=$ Physical Activity Energy Expenditure; HRAR = heart rate above rest; ambulatory status: $0=$ normal ambulation and $1=$ community ambulation.

Furthermore, T-test showed a significant difference $(p=0.02)$ for PAEE between normal (PAEE = $420 \mathrm{~J} / \mathrm{kg} / \mathrm{min}$ ) and community ambulatory children (PAEE $=319 \mathrm{~J} / \mathrm{kg} / \mathrm{min}$ ), but not between boys and girls. Therefore, ambulation level was entered as a dichotomous variable into the regression analyses. Colinearity was found between HRAR and HRAS, and thus HRAR was entered into the model. The significant models with the highest explained variance are displayed in Table 5.

\section{Discussion}

The first aim of this study was to test the validity of existing equations for predicting PAEE in children with SB using both the Actical and Actiheart accelerometry.

\subsection{Prediction models using acticounts only}

Contrary to the findings of other recent studies with healthy children [34], the AC model showed an large overestimation of the real PAEE. This overestimation was almost twice the PAEE as measured by the IC. The $\mathrm{AC}$ is sensitive to movements of the trunk and therefore the attachment at the hip may play an important factor in explaining these results. Children with SB often show excessive horizontal compensatory movements due to weakness of the hip musculature, probably leading to excessive amounts of acticounts which are only partly being accounted for in the true energy expenditure. Indeed, when looking at the AC counts in the study population $(2547.9 \pm 1029.9)$, the acticounts are higher and much more varied than in healthy children (1641 \pm 410$)$ [35]. Interestingly, acticounts are lower in community ambulatory children. This is most likely explained by the fact that they ambulate a much shorter distance than do their peers who are considered normal ambulatory [20]. The concern about the Actical not being able to register different gait patterns seems to be irrelevant in thispopulation, as reflected by the in- 
creased acticounts during walking. However, the main source of error in the prediction of PAEE seems to be the coefficient by which the acticounts predict PAEE. At the same time, the AH model using acticounts only showed a lower ICC and explained variance of only $29 \%$. AH acticounts in healthy children are again much lower (250-500 counts) [13] than in the study population $(857 \pm 394.3)$. There was only a moderate correlation between the $\mathrm{AH}$ acticounts and PAEE, leading to the hypothesis that, unlike in the case of the AC, $\mathrm{AH}$ acticounts by themselves are not good predictors of PAEE in children with SB. The pathological gait pattern in combination with the placement of the accelerometer at the chest may not be optimal.

\subsection{Prediction models using heart rate data combined with acticounts}

Except for the $\mathrm{AH}$ predictions using predicted HRAS, the other models using HRAR and true measured HRAS overestimated PAEE compared to PAEE measured with indirect calorimetry. An overestimation was not expected because children with SB have a higher cost of locomotion [18-20]. Another factor might be the increased number of acticounts, as discussed earlier, in combination with an even more so increased HRAR during normal walking. Corder et al. [13] reported a HRAR between 28 and 43, while the children in this study showed an average of 55.2 ( \pm 18.4 ; range 28-98) during the 6MWT. The absolute HR is similar to that of healthy peers [36], but with a much smaller distance covered. This then leads to activity occuring at the same HR, which in the prediction equation accounts for more PAEE, and is thus an overestimation. A recent study in children with SB showed an increased heart rate response for $\mathrm{VO}_{2}$ due to deconditioning [20]. This subsequently results in higher HR with similar PAEE, again causing an overestimation of PAEE when using HR assumptions in healthy children. Secondary database analysis showed a significant, but only moderate correlation of 0.4 between measured HRAS and the AH acticounts, but a nonsignificant correlation between HRAR and AH acticounts. Indeed, when using the model for children with chronic disease based on (predicted) HRAS as well, differences are much smaller, possibly due to similar problems of deconditiong in these children with chronic disease. Takken's model was most promising in the study population as well, with an ICC of 0.73 and the smallest LoA (still quite large); however, this model yielded an explained variance for children with SB of only $55 \%$.
Finally, looking at the models using predicted HRAS, ICC and $\mathrm{R}^{2}$ were extremely poor, which was also reflected in the lack of correlation between predicted HRAS and PAEE. T-tests also showed a large significant difference of 17.5 beats per minute and poor correlation between predicted and measured HRAS.

\subsection{New equations}

Considering the correlations between PAEE and anthropometric and physiological variables, a couple of results stand out. First, unlike in healthy children, PAEE did not decrease with age, while REE did, as expected. This is in line with earlier unpublished data from the current investigators, which includes 70 children. Whereas in healthy children PAEE during ambulation declines with age due to improved biomechanics and oxygen utilization, in children with SB, ambulation often becomes more difficult with age due to possible deconditioning and insufficient muscle power combined with increasing height and weight. Secondly, there was a significant difference in PAEE and acticounts between normal and community ambulatory children, in line with earlier results regarding energy cost of locomotion in children with SB [20,22]. Therefore, Hoffer classification and not age was entered into the models. Finally, unlike in healthy children, there were no differences between boys and girls except for skinfolds. Skin folds, just like height, weight, BMI, and predicted HRAS did not correlate with true PAEE and were therefore not included in the regression analyses.

The regression model for the Actiheart using HRAR and Hoffer classification provided the most accurate prediction model $\left(\mathrm{R}^{2}=0.720\right)$ for the PAEE in children with $\mathrm{SB}$, followed by the AC model using accelerocounts and Hoffer classification $\left(\mathrm{R}^{2}=0.681\right)$. The accelerocounts by the Actiheart were removed from the models, because they did not add significantly to the prediction in addition to the other variables. In the correlation analysis, the AH accelerocounts only showed a moderate correlation to PAEE. This seems in line with the models of Corder [12,13] and Takken [20], in which accelerocounts only account for a small part of the variation in predicted PAEE. In those models, most of the variation seems to be explained by (predicted) HR measures as well. The explained variance of the new Actiheart model was 72\%, while that of the new Actical model was 68\%. These numbers are comparable to Corder and Takken, who reported $65 \%$ (for the $\mathrm{AC}$ ) and $65 \%$ for the $\mathrm{AH}$ in children with chronic disease, but still lower than Corder's 
equations for healthy children with an explained variance of $86 \%$ for the $\mathrm{AH}$. The lower explained variance compared to healthy children could be explained by the large variability in motor function, HR, and REE within the children with SB. For example, two children with the same level of lesion can experience different limitations in motor function, walking pattern, fitness level, and energy cost of locomotion. This is also in line with the finding that adding ambulatory status improved all models.

This study had some limitations. It was conducted with ambulatory children with SB during ambulation only; the results of this study are neither applicable to the whole SB patient population nor for all activities. At the same time, it is known from this population that ambulatory activities account for most of the PAEE. For further validation of the newly derived models, the prediction equations should ideally be cross-validated in both a different group of ambulatory children with $\mathrm{SB}$ and in more activities of daily life. Another method would be to compare the models using a generalized F-test, but this is only possible when the models are "nested", which is the case when they are using the same variables. Since these new models have a disease specific variable (ambulatory status) in the model, this is not possible. The new models, however, have improved the explained variation without adding more variables into the equations. Whereas the original AC model showed a $\mathrm{R}^{2}$ of 0.61 , this was improved to 0.68 , and for the $\mathrm{AH}$, the original explained variance ranging from $7 \%$ to $65 \%$ improved to $72 \%$.

Some limitations in feasibility were noted in using the activity monitors. There were some difficulties due to malfunctioning of the Actiheart and Actical software/hardware: the data of 13 children could not be retrieved. In addition, the participants complained about the AH: the ECG electrodes resulted in irritating reactions on the skin at the place of attachment. The use of a less expensive heart rate monitor might reduce these reactions. The AC, on the other hand, was easier to attach and more user friendly.

A major strength of this study is the use of directly measured HRAS. Where several other studies have used unpublished data from Corder's study [13] to predict sleeping heart rate, this study obtained true sleeping heart rate from the three-day period during which the participants were being monitored. Corder's prediction was derived from 12- and 13-year-old healthy children, which could explain some of the differences. In this study, large differences and no correlation were found between predicted HRAS and measured HRAS or PAEE. Because of this, the use of directly measured HRAS is recommended when this is a variable of interest, instead of the predicted HRAS.

\section{Conclusion}

The present study shows that existing equations to predict PAEE using the activity monitors $\mathrm{AC}$ and $\mathrm{AH}$ are not valid for use in children with SB for the individual evaluation of PAEE. The best regression model derived during this study seems to be registration of heart rate above rest, followed by a new model for the AC, both including a variable for ambulatory status. An additional benefit of registration of heart rate above rest only would be that it does not require the use of expensive accelerometry equipment and can easily be performed by clinicians working with these patients. Cross-validation of these regression models is still needed.

\section{Acknowledgements}

The authors would like to thank the children and the parents for their participation in this study. We would also like to thank C. Kruitwagen for his statistical suggestions and Dr. T.P. Schilling for proofreading and correcting this manuscript. This study was funded by the Dutch Royal Society for Physiotherapy, the Wilhelmina Children's Hospital Research Fund, Stichting BIO-Kinderrevalidatie and SIA-RAAK.

\section{Conflict of interest}

The authors have reported no conflict of interest.

\section{References}

[1] International Clearinghouse for Birth Defects Surveillance and Research. Annual Report of 2011 with Data for 2009. [Cited 3-12-2012] Available from URL: http://www.icbdsr. org/filebank/documents/ar2005/Report2011.pdf.

[2] K.D. Ryan, C. Ploski and J.B. Emans, Myelodysplasia - the musculoskeletal problem - habilitation from infancy to adulthood, Physical Therapy 71 (1991), 935-946.

[3] R.M. Bowman, D.G. McLone, J.A. Grant, T. Tomita and J.A. Ito, Spina bifida: A 25-year prospective, Pediatr Neurosurg 34 (2001), 114-120.

[4] D.K. Singh, Families of children with spina bifida: A review, Journal of Developmental and Physical Disabilities 15 (2003), 37-54.

[5] L.E. Mitchell, N.S. Adzick, J. Melchionne, P.S. Pasquariello, L.N. Sutton and A.S. Whitehead, Spina bifida, Lancet 364 (2004), 1885-1895. 
[6] M.E. Roebroeck, R. Jahnsen, C. Carona, R.M. Kent and M.A. Chamberlain, Adult outcomes and lifespan issues for people with childhood-onset physical disability, Dev Med Child Neurol 51 (2009), 670-678.

[7] R.J. Simeonsson, J.S. McMillen and G.S. Huntington, Secondary conditions in children with disabilities: Spina bifida as a case example, Mental Retardation and Developmental Disabilities Research Reviews 8 (2002), 198-205.

[8] H.J.G. van den Berg-Emons, J.B.J. Bussmann, H.J. Meyerink, M.E. Roebroeck and H.J. Stam, Body fat fitness and level of everyday physical activity in adolescents and young adults with meningomyelocele, Journal of Rehabilitation Medicine 35 (2003), 271-275.

[9] L.M. Buffart, M.E. Roebroeck, M. Rol, H.J. Stam and R.J.G. van den Berg-Emons, Triad of physical activity aerobic fitness and obesity in adolescents and young adults with myelomeningocele, Journal of Rehabilitation Medicine $\mathbf{4 0}$ (2008), 70-75.

[10] M.A.G.C. Schoenmakers, J.F. De Groot and J.W. Gorter, Muscle strength aerobic capacity and physical activity in independent ambulating children with lumbosacral spina bifida, Disability \& Rehabilitation 31 (2009), 259-266.

[11] R.A. Littlewood, O. Trocki, R.W. Shepherd, K. Shepherd and P.S.W. Davies, Resting energy expenditure and body composition in children with myelomeningocele, Developmental Neurorehabilitation 6 (2003), 31-37.

[12] B.H. Dobkin and A. Dorsch, The promise of mHealth: Daily activity monitoring and outcome assessments by wearable sensors, Neurorehabil Neural Repair 25(9) (Nov-Dec 2011), 788-798.

[13] K. Corder, S. Brage, N.J. Wareham and U. Ekelund, Comparison of PAEE from combined and separate heart rate and movement models in children, Medicine and Science in Sports and Exercise 37 (2005), 1761-1767.

[14] K. Corder, S. Brage, C. Mattocks, A. Ness, C. Riddoch, N.J. Wareham and U. Ekelund, Comparison of two methods to assess PAEE during six activities in children, Med Sci Sports Exerc 39(12) (Dec 2007), 2180-2188.

[15] S.M. de Graauw, J.F. de Groot, M. van Brussel, M.F. Streur and T. Takken, Review of prediction models to estimate activity-related energy expenditure in children and adolescents, Int J Pediatr 2010 (2010), 489304.

[16] D.R. Bassett, B.E. Ainsworth, A.M. Swartz, S.J. Strath, W.L. O'Brien and G.A. King, Validity of four motion sensors in measuring moderate intensity physical activity, Medicine and Science in Sports and Exercise 32 (2000), S471-S480.

[17] S.E. Crouter, J.R. Churilla and D.R. Bassett, Accuracy of the actiheart for the assessment of energy expenditure in adults, European Journal of Clinical Nutrition 62 (2008), 704-711.

[18] C.M. Duffy, A.E. Hill, A.P. Cosgrove, I.S. Corry and H.K. Graham, Energy consumption in children with spina bifida and cerebral palsy: A comparative study, Developmental Medicine and Child Neurology 38 (1996), 238-243.

[19] A. Bare, S.J. Vankoski, L. Dias, M. Danduran and S. Boas, Independent ambulators with high sacral myelomeningocele: The relation between walking kinematics and energy consumption, Developmental Medicine and Child Neurology $\mathbf{4 3}$ (2001), 16-21.

[20] J.F. de Groot, T. Takken, M.A. Schoenmakers, L. Vanhees and P.J. Helders, Limiting factors in peak oxygen uptake and the relationship with functional ambulation in ambulating children with spina bifida, Eur J Appl Physiol 104(4) (Nov 2008), $657-665$.
[21] T. Takken, S. Stephens, A. Balemans, M.S. Tremblay, D.W. Esliger, J. Schneiderman, D. Biggar, P. Longmuir, V. Wright, B. McCrindle, M. Hendricks, A. Abad, J. van der Net, J. Beyene and B.M. Feldman, Validation of the actiheart activity monitor for measurement of activity energy expenditure in children and adolescents with chronic disease, Eur J Clin Nutr 64(12) (Dec 2010), 1494-1500.

[22] J.F. de Groot, T. Takken, M. van Brussel et al., Randomized controlled study of home-based treadmill training for ambulatory children with spina bifida, Neurorehabilitation and Neural Repair 25 (2011), 597-606.

[23] M.M. Hoffer, E. Feiwell, R. Perry, J. Perry and C. Bonnett, Functional ambulation in patients with myelomeningocele, Journal of Bone and Joint Surgery-American A 55 (1973), 137-148.

[24] M.A.G.C. Schoenmakers, C.S.P.M. Uiterwaal, V.A.M. Gulmans, R.H.J.M. Gooskens and P.J.M. Helders, Determinants of functional independence and quality of life in children with spina bifida, Clinical Rehabilitation 19 (2005), 677-685.

[25] M.L. Pollack, D.H. Schmidt and A.S. Jackson, Measurement of cardio-respiratory fitness and body composition in the clinical setting, Comprehensive Therapy 6 (1980), 12.

[26] J.I. Medbo, A. Mamen, B. Welde, E. Von Heimburg and R. Stokke, Examination of the metamax I and II oxygen analysers during exercise studies in the laboratory, Scandinavian Journal of Clinical \& Laboratory Investigation 62 (2002), 585-598.

[27] M.A. Brehm, J. Harlaar and H. Groepenhof, Validation of the portable VmaxST system for oxygen-uptake measurement, Gait \& Posture 20 (2004), 67-73.

[28] J.F. De Groot, T. Takken, M.A.G.C. Schoenmakers, L. Tummers, L. Vanhees and P.J.M. Helders, Reproducibility of energy cost of locomotion in ambulatory children with spina bifida, Gait \& Posture 31 (2010), 159-163.

[29] ATS statement: Guidelines for the six-minute walk test, Am J Respir Crit Care Med 166 (2002), 111-117.

[30] L. Garby and A. Astrup, The relationship between the respiratory quotient and the energy equivalent of oxygen during simultaneous glucose and lipid oxidation and lipogenesis, Acta Physiologica Scandinavica 129 (1987), 443-444.

[31] S. Brage, N. Brage, P.W. Franks, U. Ekelund and N.J. Wareham, Reliability and validity of the combined heart rate and movement sensor actiheart, European Journal of Clinical Nutrition 59 (2005), 561-570.

[32] L.G. Portney and M.P. Watkins, Foundations of clinical research: Applications to practice, Prentice Hall, Upper Saddle River, NJ, 2009.

[33] J.M. Bland and D.G. Altman, Statistical methods for assessing agreement between two methods of clinical measurement lancet i (1986), View Record in Scopus, Cited By in Scopus (14215) (1986), 307-310.

[34] S. Alhassan, K. Lyden, C. Howe, S.K. Keadle, O. Nwaokelemeh and P.S. Freedson, Accuracy of accelerometer regression models in predicting energy expenditure and METs in children and youth, Pediatr Exerc Sci 24(4) (2012), 519-536.

[35] M.R. Puyua, A.L. Adolph, F.A. Vohra, I. Zakeri and N.F. Butte, Prediction of activity energy expenditure using accelerometers in children, Medicine \& Science in Sports \& Exercise Issue 36(9) (2004), 1625-1631.

[36] A.M. Li, J. Yin, J.T. Au, H.K. So, T. Tsang, E. Wong, T.F. Fok and P.C. Ng, Standard reference for the six-minute-walk test in healthy children aged 7 to 16 years, American Journal of Respiratory and Critical Care Medicine 176 (2007), 174-180. 